\title{
Alívio da dor e sofrimento psicológico em oncologias pediátricas: uma revisão sistemática
}

RESUMO | Objetivo: identificar intervenções para reduzir dor e sofrimento psicológico nas oncologias pediátricas durante procedimentos com agulhas. Método: Conduziu-se uma revisão sistemática entre novembro de 2020 e maio de 2021 nas bases MEDLINE e PsycINFO. Foram inclusos: estudos primários com intervenções comportamentais e/ou farmacológicas sem restringir idioma ou ano de publicação e exclusos: secundários e duplicatas. Resultados: Após a elegibilidade, 26 estudos formaram a síntese. O contato familiar, palhaçadas médicas, desviar o olhar, musicoterapia, realidade virtual e hipnose foram as principais técnicas psicológicas. Foram considerados eficazes os métodos farmacológicos: fentanil com etomidato, dose baixa oral ou spray nasal de midazolam e xarope de acetaminofeno. A associação de lidocaína e prilocaína com hipnose mostrou-se efetiva, assim como, midazolam associado com jogos de vídeo ou livros musicais. Conclusão: O caminho para a reduzir dor e sofrimento psicológico é um processo complexo e pode haver um sinergismo entre intervenções psicológicas e farmacológicas.

Palavras-chaves: Assistentes de Pediatria"; Pediatria; Câncer

\begin{abstract}
Objective: to identify interventions to reduce pain and psychological distress in pediatric oncology patients during needle procedures. Method: A systematic review was conducted between November 11, 2020 and May 17, 2021 in MEDLINE and PsycINFO. We included: primary studies with behavioral and/or pharmacological interventions without restricting language or year of publication and excluded: secondary and duplicates. Results: After eligibility, 26 studies formed the synthesis. Family contact, medical clowning, looking away, music therapy, virtual reality and hypnosis were the main psychological techniques. Pharmacological methods were considered effective: fentanyl with etomidate, low dose oral or nasal spray of midazolam, and acetaminophen syrup. The combination of lidocaine and prilocaine with hypnosis was shown to be effective in reducing fear and pain, as well as midazolam associated with video games or music books. Conclusion: The way to reduce psychological pain and suffering is a complex process and there may be a synergism between psychological and pharmacological interventions.
\end{abstract}

Keywords: Pediatric Assistants", Pediatrics; Cancer.

RESUMEN | Objetivo: Identificar intervenciones para reducir el dolor y la angustia psicológica en los pacientes de oncología pediátrica durante los procedimientos con agujas. Método: Se realizó una revisión sistemática entre el 11 de noviembre de 2020 y el 17 de mayo de 2021 en MEDLINE y PsycINFO. Se incluyeron: estudios primarios con intervenciones conductuales y/o farmacológicas sin restringir el idioma o el año de publicación y se excluyeron: secundarios y duplicados. Resultados: Tras la elegibilidad, 26 estudios formaron la síntesis. El contacto con la familia, la payasada médica, la mirada ausente, la musicoterapia, la realidad virtual y la hipnosis fueron las principales técnicas psicológicas. Se consideraron eficaces los métodos farmacológicos: fentanilo con etomidato, dosis bajas de midazolam por vía oral o nasal y jarabe de paracetamol. La unión de lidocaína y prilocaína con hipnosis resultó eficaz para reducir el miedo y el dolor, así como el midazolam asociado a videojuegos o libros musicales. Conclusión: La forma de reducir el dolor y el sufrimiento psicológico es un proceso complejo y puede haber un sinergismo entre las intervenciones psicológicas y farmacológicas.

Palabras claves: Asistentes de Pediatría", Pediatría; Cáncer

\begin{abstract}
Regina Petrola Bastos Rocha
Enfermeira, Pós Graduada em Saúde Pública e em Gestão dos Sistemas e serviços de Saúde (Gestão do SUS), Mestre em Desenvolvimento Regional Sustentável e Doutoranda em Ciências da saúde. Docente nos cursos de Enfermagem e Medicina da Faculdade de Medicina Estácio de Juazeiro do Norte - Estácio FMJ. ORCID: 0000-0003-0626-232X
\end{abstract}

\section{Raimundo Monteiro da Silva Neto}

Graduação em enfermagem em andamento pelo Centro Universitário de Juazeiro do Norte (UNIJUAZEIRO), Juazeiro do Norte (CE), Brasil.

ORCID: 0000-0001-9949-1740

\section{Camila Bezerra Silva}

Enfermeira, graduada na Faculdade de Medicina Estácio de Juazeiro do Norte. Pós-graduanda em Urgência e Emergência pela Universidade Regional do Cariri. Plantonista da Emergência no Hospital Unimed Cariri.

ORCID: 0000-0003-0070-9434

\section{Vanessa Ruth Ferreira da Silva}

Discente do curso de enfermagem no Centro Universitário de Juazeiro do NorteUNIJUAZEIRO.

ORCID: 0000-0002-7388-3102

\section{Maria Daniely da Silva Santos Souza}

Graduação em enfermagem em andamento pelo Centro Universitário de Juazeiro do Norte (UNIJUAZEIRO), Juazeiro do Norte (CE), Brasil.
ORCID: 0000-0003-1416-2900

\section{Cíntia de Lima Garcia}

Enfermeira. Doutorado em Ciências com ênfase em Saúde Coletiva (2019) Centro Universitário Saúde $A B C$, FMABC, Santo André (SP), Brasil. Docente dos cursos de Medicina e Enfermagem da Faculdade de Medicina Estácio de Juazeiro do Norte (ESTACIO FMJ). Coordenadora do Laboratório de Habilidades e Simulação Realística (LHS) da ESTACIO FMJ. Coordenadora de período do curso de Medicina da ESTACIO FM Juazeiro do Norte (CE).

ORCID: 0000-0003-2673-7003

Recebido em: 24/06/2021

Aprovado em: 23/07/2021 


\section{INTRODUÇÃO}

Um diagnóstico neoplásico pode representar uma grande perturbação na vida cotidiana, principalmente das crianças 1. A incidência de câncer em crianças durante os três próximos anos no Brasil é de 4.310 casos novos para crianças do sexo masculino e de 4.150 para as do sexo feminino 2. Elas encontram-se em um mundo onde adultos, conhecidos ou não, realizam praticamente todas as tomadas decisões 1 .

A maioria dessas crianças que recebem tratamentos em oncologias pediátricas necessitam de algum procedimento que podem gerar medo ou dor 3. Normalmente eles experimentam níveis crescentes de ansiedade e não adaptam-se com facilidade ao desconforto causado por procedimentos invasivos com agulhas 1. Com frequência, desenvolvem sintomas como inapetência, insônia e sofrimento psicológico que precedem suas visitas hospitalares 4.

A sistematização da assistência de enfermagem (SAE) em oncologias pediátricas é complexa e engloba as diferentes etapas do cuidar. Desde ações preventivas até os tratamentos prolongados, farmacológicos ou não 5. Por relatam uma série de aspectos negativos e fatores relacionados à sua experiência de doença, necessitam de uma maior diversidade de intervenções farmacológicas e/ou comportamentais 6 . Alguns procedimentos como inserção acesso venoso central e punções lombares podem ser cúmplices da angústia, do medo e dor 7 .

A prestação de cuidados ideais a pacientes oncológicos é uma prioridade de saúde, mas, sabe-se relativamente pouco sobre o que pacientes, famílias e provedores de saúde consideram como "melhores" práticas para promoção do bem-estar do paciente 8 . A comunicação e as experiências dos pacientes oncológicos pediátricos com os profissionais de saúde são comple- tamente diferentes das dos adultos 9 . Pacientes em oncologias pediátricas correm alto risco de deterioração psicossomática e necessitam de cuidados de alta qualidade 10 .

Torna-se necessário que o cuidado seja sistematizado de uma forma que contemple as necessidades das crianças, diante disso surgiu a pergunta norteadora que foi estruturada na estratégia de busca população variável outcome/ desfecho (PVO): A assistência pode ser sistematizada com intervenções comportamentais e farmacológicas, de modo que, reduza agravos psicológicos e dor em crianças com câncer durante procedimentos invasivos?

O estudo objetivou identificar intervenções que possam ser utilizadas para reduzir dor e sofrimento psicológico em oncologias pediátricas durante procedimentos com agulhas.

\section{MÉTODO}

Foi conduzida uma revisão sistemática de acordo com as recomendações metodológicas do Preferred Reporting Items for Systematic Reviews and Meta -Analyses (PRISMA) 12.

A pergunta de partida deu-se através da estratégia de busca PVO, isto é, P (População), V (Variável) e O (Outcome/Desfecho) 13. No estudo: $\mathrm{P}=\mathrm{Pa}-$ cientes em oncologias pediátricas, $\mathrm{V}=$ Intervenções comportamentais e farmacológicas e $\mathrm{O}=$ Redução de dor e/ ou sofrimento psicológico. Os critérios de inclusão estabelecidos foram: a) Ser um estudo primário, b) Mostrar resultados de intervenções comportamentais e farmacológicas e c) sem restringir idioma ou ano de publicação. Foram exclusos: I) Estudos secundários, II) duplicatas, checadas através do gerenciador de referências EndNote versão X5.

As buscas ocorreram entre os dias 11 de novembro de 2020 e 17 maio de 2021 e nas bases de dados da MEDLINE e PsycINFO com os MeSH termos: "Nurses, Pediatric", "Pediatric Assis- tants", Pediatrics e Cancer. A principal estratégia de busca utilizada foi: "Pediatric AND cancer AND nurse". O processo de elegibilidade ocorreu através de dois revisores de maneira individual e iniciou-se através da leitura de títulos, posteriormente, resumos e a terceira etapa na integra. Após a leitura na íntegra, alguns estudos foram exclusos pois não abordavam a temática: "oncologia e/ou pediátrica". Quando houve alguma dúvida de inclusão/exclusão a orientadora foi consultada para contribuir com a resolução da dúvida, se o estudo entraria ou não na revisão.

A extração de dados ocorreu com o auxílio de uma matriz para expor os principais resultados e criar uma tabela síntese, onde foram extraídos os dados: Autor, ano, país, métodos e desfechos. Os resultados foram divididos em três categorias: Intervenções psicológicas, Intervenções farmacológicas e intervenções psicológicas e farmacológicas.

A evidência foi avaliada através do sistema Desenvolvimento e Avaliação da Classificação de Recomendações (GRADE) e o estudo focou principalmente em intervenções psicológicas e farmacológicas. A abordagem de Avaliação do sistema GRADE fornece um sistema para classificar a qualidade das evidências. A qualidade é classificada em quatro: alta, moderada, baixa e muito baixa. A força das recomendações que é explícito, abrangente, transparente e pragmático, classificado em: forte e fraca. E está cada vez mais sendo adotado por organizações em todo o mundo 14.

O risco de viés foi avaliado usando a ferramenta da Risk of Bias figura 1 e figura 2 da Colaboração Cochrane 15 . Esta ferramenta avalia o risco de viés de seleção), viés de desempenho, viés de detecção, viés de atrito), viés de relatórios e outros vieses. Cada ensaio clínico foi avaliado nestes sete critérios, eles foram classificação como "baixo risco" (Low risk), "alto risco" (High risk), ou "pouco claro" (Unclear risk). 


\section{RESULTADOS}

Após realizar as buscas nos bancos de dados foram encontrados incialmente 1.429 estudos e na etapa de elegibilidade 135 manuscritos foram lidos na íntegra. Destes, 26 estudos foram inclusos na síntese final deste estudo e estão dispostos no fluxograma Prisma 2009 Figura 312.

Foi evidenciado que a musicoterapia, óculos de realidade virtual, hipnose, palhaçadas médicas podem contribuir na redução da dor. Os anestésicos locais como o fentanil combinado com etomidato e spray nasal midazolam são úteis para oferecer alívio nos pro-

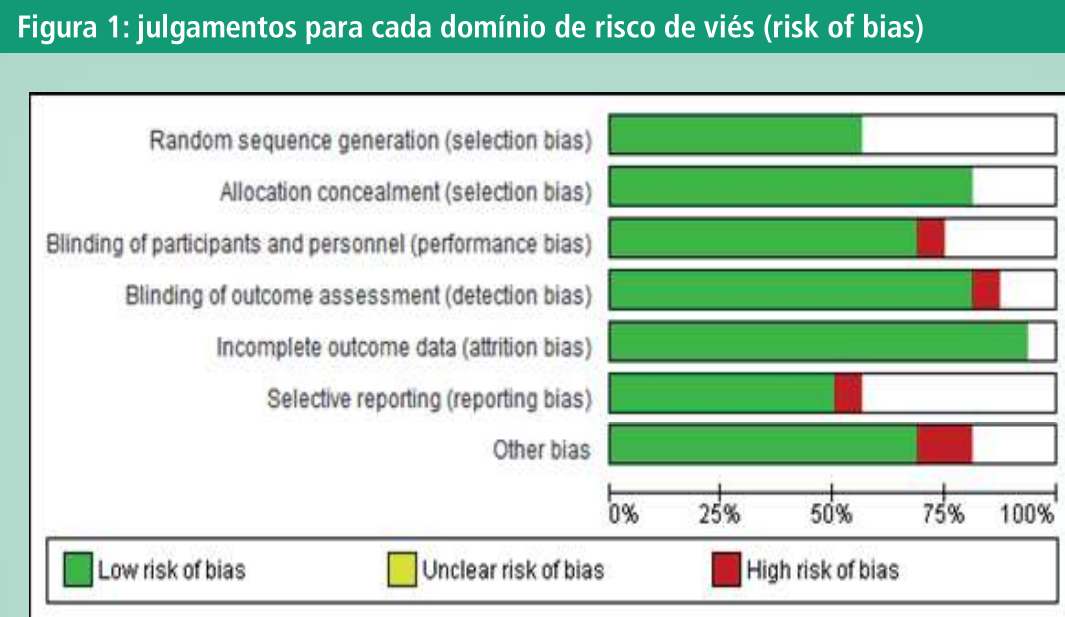

Fonte: Autora, 2021

Figura 2: A qualidade metodológica dos estudos incluídos com base nos julgamentos dos autores da revisão sobre cada item de risco de viés apresentado como porcentagens em todos os estudos incluidos (risk of bias)

\begin{tabular}{|c|c|c|c|c|c|c|c|c|c|c|c|c|c|c|c|c|}
\hline 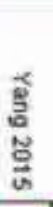 & 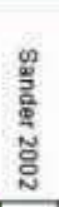 & 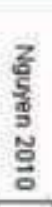 & 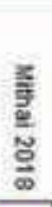 & 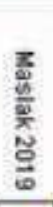 & 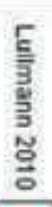 & 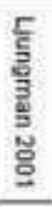 & 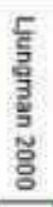 & 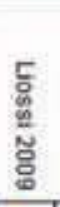 & 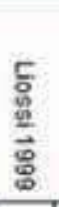 & $\frac{\mathrm{K}}{\mathrm{E}}$ & 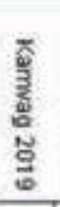 & 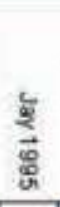 & 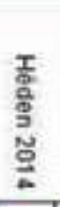 & 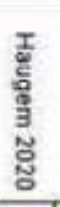 & 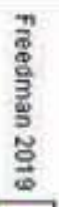 & \\
\hline (c) & & & (6) & 4 & (4) & & & 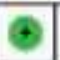 & & & 4 & & $\theta$ & 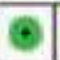 & (4) & Random sequence generation (selection bias) \\
\hline & $\oplus$ & $\oplus$ & $\ominus$ & $\odot$ & $\oplus$ & $\odot$ & $\oplus$ & & $\oplus$ & $\bullet$ & 4 & $\oplus$ & & $\theta$ & $\oplus$ & Aliocation concealment (selection bias) \\
\hline (c) & $\theta$ & $\oplus$ & & & & - & (๑) & 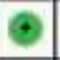 & • & 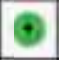 & (†) & & c & 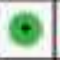 & $\oplus$ & Blinting of participants and personnel (performance bias) \\
\hline$\theta$ & & (당 & 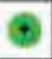 & c & $\oplus$ & • & • & $\theta$ & $\bullet$ & $\bullet$ & • & & $\bullet$ & $\odot$ & $\oplus$ & Binding of outcome assessment (detection bias) \\
\hline (6) & $\theta$ & (4) & (5) & (†) & (૯) & e & () & (†) & () & (4) & $\oplus$ & $\oplus$ & & c) & (†) & Incomplete outcome data (atrition bias) \\
\hline () & & • & & () & 두 & (2) & & & & & • & • & $\bullet$ & e & & Selective reporting (reporting bias) \\
\hline$\theta$ & $\oplus$ & (ㄷ) & ( & & 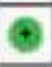 & 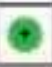 & (c) & $\theta$ & (3) & • & & $\theta$ & & $\bullet$ & (4) & Other bias \\
\hline
\end{tabular}

Fonte: Autora, 2021

cedimentos com agulhas. A lidocaína associada com prilocaína e hipnose mostraram-se efetivas, assim como, o midazolam com jogos de vídeo e livros musicais.

\section{DISCUSSÃo}

A pesquisa evidenciou a partir de estudos observacionais e clínicos que métodos comportamentais e farmaco- lógicos podem auxiliar no alívio da dor em procedimentos com agulhas em oncologias pediátricas. Métodos não farmacológicos podem ser uma alternativa ou um complemento dos analgésicos. A musicoterapia pode contribuir na redução da dor, os participantes do estudo de Nguyen et al. (2010) foram designados aleatoriamente para um grupo musical e grupo controle e as crianças do grupo musical mostraram menores índices de dor e ansiedade durante e após a punção lombar 23.

Em Sander et al (2020) foi estudado outra intervenção não farmacológica em crianças com câncer submetidos a punção lombar. Ele Realizou intervenção padrão durante a punção, mas o grupo experimental também usou óculos de realidade virtual e assistiu a um vídeo e todos os escores foram mais baixos no grupo que utilizou ócu- 


\section{Figura 3. Diagrama de Fluxo PRISMA}

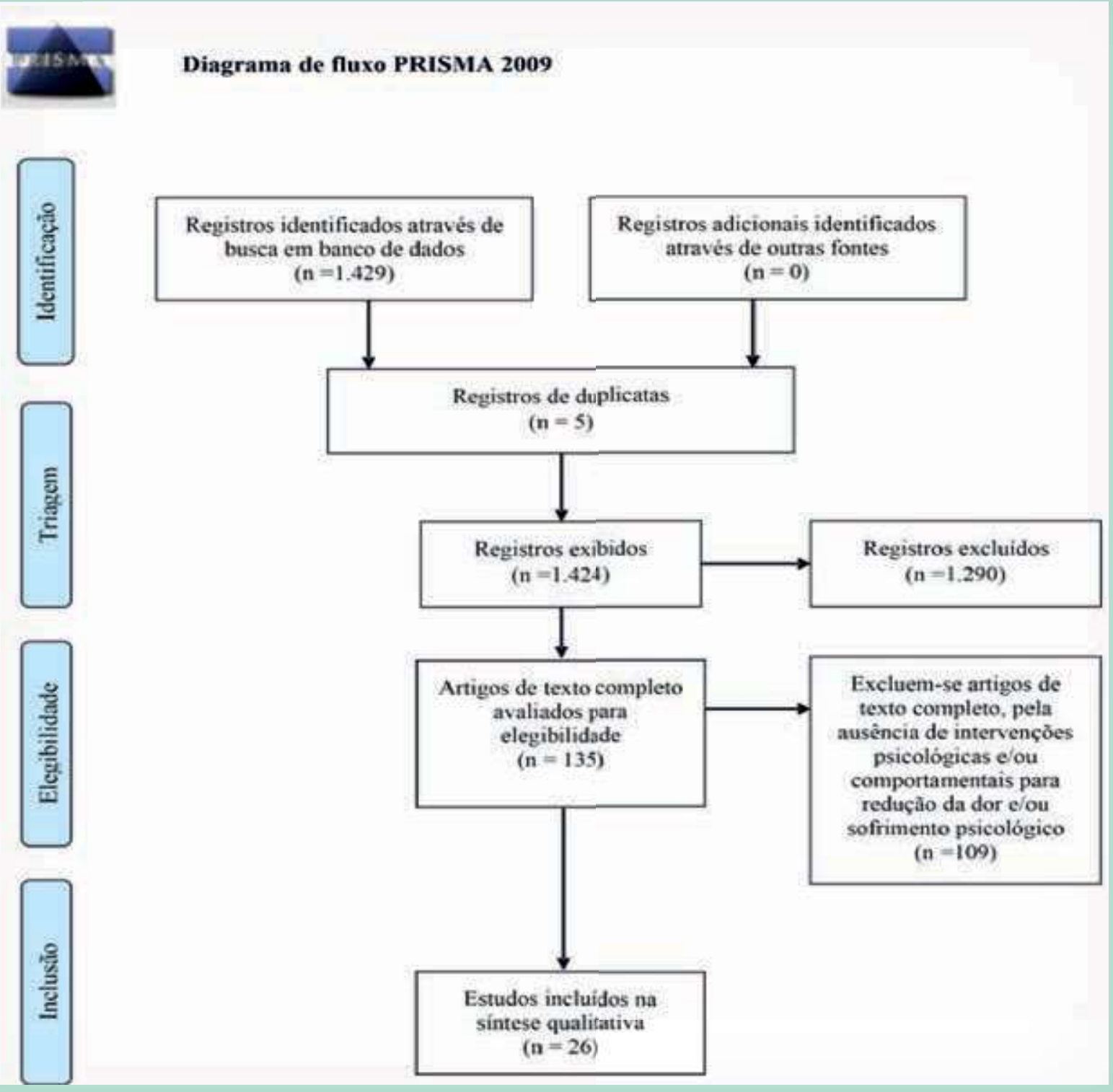

Fonte: Autora, 2021

los de realidade virtual 27. Há pouco mais de duas décadas, Liossi realizou um estudo para comparar a eficácia da hipnose clínica com o treinamento de habilidades cognitivas comportamentais para aliviar a dor e o sofrimento de pacientes com câncer pediátrico. Os resultados mostraram que a hipnose pode ser uma boa escolha para reduzir o sofrimento psicológico 30.

Anestésicos locais tópicos proporcionam analgesia eficaz para pacientes submetidos a inúmeros procedimentos 43. Para Lullmann et al. (2010) o alívio da dor pode ser sinônimo de qualidade de vida para as crianças com câncer que necessitam de procedimentos com agulhas. Ele realizou pesquisa, onde, após a aplicação do creme de lidocaína-prilocaína (EMLA) as crianças do grupo intervenção obtiveram uma redução significativa da dor após $60 \mathrm{~min}$ de tempo de aplicação. Mostrou que anestésicos locais podem ajudar no alívio da dor durante procedimentos com agulhas 22 .

O estudo de Hedén et al. (2009), 
Tabela 1. Descrição dos resultados selecionados:Autor, ano, país, desfechos e classificação do nível de evidência dos ensaios clínicos randomizados

AUTOR, ANO PAÍS

KAMSVÅG et al., $2020^{16}$ Suécia

HAUGEN et al., $2020^{17}$ EUA

FREEDMAN et al., $2019^{18}$ Canada
DESFECHOS

CNE

O ibuprofeno mostrou-se pouco útil na redução de medo e dor com procedimentos com agulha. Não podemos afirmar que o ibuprofeno não é útil em procedimentos com agulhas, mas que parece improvável.

Compromisso da equipe com o bem-estar dos pacientes, prática é aprimorada quando os enfermeiros.

0 grupo intervenção (enfrentamento) tinham maior conhecimento, menos medo e maior disposição para a aceitação com procedimentos envolvendo agulhas após a sessão de educação em sala de aula.

Oferecer às crianças a escolha do suporte médico durante procedimentos invasivos permite um suporte personalizado baseado nas necessidades individuais e é uma modalidade eficaz para devolver o controle ativo aos pacientes jovens, limitando o trauma emocional do câncer e o tratamento.

MASLAK et al., 2019 China

Aconselhar as pessoas a desviar o olhar da agulha reduz o medo e reservar um tempo junto com a criança.

MITHAL et al., $2018^{19}$ Canada

YANG et al., $2015^{20}$ China

HEDÉN; VON ESSEN; LUNGMAN, 2014 Suécia
É seguro e eficaz aplicar fentanil combinado com etomidato para sedação e analgesia.

$\oplus \oplus \oplus$

0 acetaminofeno reduziu o sofrimento autoavaliado e o sofrimento comportamental. 0 paracetamol não ofereceu nenhum efeito aditivo na redução da dor, medo e angústia quando combinado com anestesia tópica em crianças submetidas à inserção de agulha.

LÜLLMANN et al., $2010^{22}$ Alemanha

NGUYEN et al., $2010^{23}$ Vietnã

(HEDÉN et al., $2009^{24}$ Suécia
Os resultados mostraram menores índices de dor e frequência cardíaca e respiratória no grupo de música durante e após a punção lombar.

A dose baixa de midazolam oral foi eficaz para reduzir o medo e a angústia em pacientes oncológicos pediátricos. Especialmente em crianças menores, submetidos à inserção subcutânea de agulha.

LIOSSI; WHITE; HATIRA, $2009^{25}$ Reino Unido
Pacientes do grupo anestésico local mais hipnose relataram menos ansiedade antecipada, e menos dor e ansiedade relacionados ao procedimento.
ANNALFI et al., $2005^{26}$ EUA
Os efeitos das técnicas não-farmacológicas sobre a ansiedade foram percebidos muito positivamente tanto pelas crianças quanto pelos pais. 0 estudo sugere que sedação moderada se compara favoravelmente à anestesia geral no que diz respeito tanto à segurança quanto à eficácia.
SANDER WINT et al., $2002^{27}$ EUA

LUNGMAN et al., 200128 EUA
Os óculos realidade virtual são um coadjuvante viável, apropriado para a idade e não-farmacológico dos cuidados convencionais no manejo da dor associada com a punção lombar em adolescentes 


\begin{tabular}{|c|c|c|}
\hline $\begin{array}{l}\text { LUNGMAN et al., } 2000^{29} \\
\text { EUA }\end{array}$ & $\begin{array}{c}\text { A sedação consciente é indicada quando outros meios para superar o medo de uma criança } \\
\text { falham. Os pais e enfermeiros relataram redução da ansiedade, desconforto e problemas de pro- } \\
\text { cedimento para crianças do grupo de midazolam e prefeririam o mesmo medicamento no próximo } \\
\text { procedimento. Eles também relataram redução da dor. O desconforto nasal foi o efeito colateral } \\
\text { mais comum. }\end{array}$ & $\oplus \oplus \oplus$ \\
\hline $\begin{array}{l}\text { LIOSSI; HATIRA, } 1999^{30} \\
\quad \text { Reino Unido }\end{array}$ & $\begin{array}{l}\text { Os pacientes que receberam ou hipnose relataram menos dor e ansiedade relacionada à dor do } \\
\text { que os pacientes controlados e menos dor e ansiedade do que em sua própria linha de base. A } \\
\text { hipnose e treinamentos comportamentais são eficazes na preparação de pacientes oncológicos } \\
\text { pediátricos para procedimentos com agulhas. }\end{array}$ & $\oplus \oplus \oplus$ \\
\hline $\begin{array}{l}\text { KAZAK et al., } 1996^{31} \\
\text { EUA }\end{array}$ & $\begin{array}{l}\text { As classificações das mães e enfermeiras quanto à angústia infantil indicavam menos angústia } \\
\text { infantil no grupo de intervenção farmacológica associada a intervenção psicológica. }\end{array}$ & $\oplus \oplus \oplus$ \\
\hline $\begin{array}{l}\text { JAY et al., } 1995^{32} \\
\text { EUA }\end{array}$ & $\begin{array}{l}\text { Os pais classificaram significativamente mais sintomas de ajuste comportamental } 24 \mathrm{~h} \text { após serem } \\
\text { submetidos a aspirações de medula óssea quando seus filhos receberam anestesia. }\end{array}$ & $\oplus \oplus \oplus$ \\
\hline $\begin{array}{l}\text { CNE = CLASSIFICAÇÃO DO NÍVEL DA E } \\
++++=\text { Alta } \\
+++=\text { Moderada } \\
++=\text { Baixa } \\
+=\text { Muito baixa }\end{array}$ & & \\
\hline
\end{tabular}

Tabela 2. Descrição dos resultados selecionados: Autor, ano, país, desfechos e classificação do nível de evidência dos estudos transversais

AUTOR, ANO PAÍS

GRAETZ et al., 2020a ${ }^{10}$

EUA

\section{MEKONNEN; GEBREYOHANNIS; CHE- $\mathrm{RIE}, 2020^{33}$ Etiópia}

KOSIR et al., $2020^{6}$ Reino Unido

GOMBERG et al., $2020^{34}$ |srael

CHEN et al., $2020^{35}$ China

\section{DESFECHOS}

A tecnologia e a automação resultaram em falta de comunicação e a compreensão é fundamental para otimizar e melhorar os resultados da assistência em oncologias pediátricas.

Os enfermeiros devem detectar precocemente para reduzir o risco de sofrimento psicológico.

Adaptação e recuperação psicológica dos jovens pode resultar em otimização do atendimento de pacientes em oncologias pediátricas

Muitos benefícios anteriormente inéditos dos palhaços médicos foram descritos. Estes benefícios relatados incluíram medidas de redução de custos para o hospital, aumento na eficiência do pessoal, melhores resultados para os pacientes e menos estresse no pessoal da equipe e redução da tristeza.

O estabelecimento da confiança e o apoio de enfermeiras, médicos, psicólogos e assistentes sociais conduzirá a prontidão das mães para lidar com o cuidado de seu filho doente. É sugerido também o aumento do tempo de visita para o apoio dos pais às crianças hospitalizadas.

Os enfermeiros devem detectar precocemente a depressão em pacientes oncológicos pediátricos. RIE, $2020^{33}$ Etiópia

SULLIVAN et al., $2020^{36}$ Canada

Atender as necessidades visíveis das crianças na oncologia pediátrica.

GRAETZ et al., 2020b ${ }^{37}$ EUA

0 trabalho em equipe foi um fator contribuinte na redução da dor e de agravos psicológicos.

Fonte: Autora, 2021

crianças estavam sendo tratadas em um ambiente de oncologia e hematologia pediátrica. Todas as crianças foram submetidas a uma inserção de agulha no estudo e foi administrado midazolam e o outro grupo recebeu placebo e o medo foi significantemente menor no grupo midazolam. Em outro estudo
CNE com o midazolam, desta vez com a administração nasal de spray. As crianças, pais e enfermeiros preencheram um questionário em escala analógica visu- 


\begin{tabular}{|c|c|}
\hline INTERVENÇÕES & FR \\
\hline Contato frequente com familiares ${ }^{35}$ & Forte \\
\hline Diminuir o tempo de permanência hospitalar ${ }^{7}$ & Forte \\
\hline Atentar-se aos sintomas de sofrimento mental ${ }^{33}$ & Fraca \\
\hline Palhaçadas médicas ${ }^{34}$ & Forte \\
\hline União de intervenções psicológicas ${ }^{6}$ & Forte \\
\hline Melhorar a comunicação interdisciplinar ${ }^{10}$ & Fraca \\
\hline Trabalho em equipe ${ }^{37}$ & Fraca \\
\hline Desviar o olhar da agulha para reduzir o medo ${ }^{19}$ & Forte \\
\hline Uso de distração ativa para todos os procedimentos com agulhas ${ }^{38}$ & Fraca \\
\hline Musicoterapia durante e após a procedimento com agulha ${ }^{23}$ & Forte \\
\hline Auto-hipnose/hipnose para reduzir a dor $25,39,40,30$ & Forte \\
\hline Distrair a criança na oncologia para reduzir o medo 39,41 & Forte \\
\hline Uso de óculos de realidade virtual ${ }^{27}$ & Fraca \\
\hline $\begin{array}{c}\text { Combinar intervenções psicológicas com intervenções farmacológicas } \\
\text { durante todo procedimento com agulhas }{ }^{31}\end{array}$ & Forte \\
\hline
\end{tabular}

Tabela 4. intervençôes farmacológicas identificadas nos estudos

\begin{tabular}{|c|c|}
\hline INTERVENÇÕES & FR \\
\hline $\begin{array}{l}\text { Anestesia local para ajudar no alívio da dor durante procedimentos com } \\
\qquad \text { agulhas }^{22}\end{array}$ & Forte \\
\hline Sedação moderada ${ }^{26}$ & Forte \\
\hline $\begin{array}{l}\text { Administração de fentanil combinado com etomidato para sedação e } \\
\text { analgesia } 20\end{array}$ & Fraca \\
\hline Sedação consciente ${ }^{28}$ & Forte \\
\hline $\begin{array}{l}\text { A dose baixa de midazolam oral para reduzir o medo e a angústia duran- } \\
\text { te os procedimentos com agulhas em crianças com câncer }{ }^{24}\end{array}$ & Fraca \\
\hline $\begin{array}{l}\text { Spray nasal midazolam para oferecer alívio nos procedimentos com } \\
\qquad \text { agulha }{ }^{29}\end{array}$ & Fraca \\
\hline 0 acetaminofeno para reduzir sofrimento comportamental ${ }^{21}$ & Fraca \\
\hline Não utilizar morfina oral para proporciona redução adicional de medo 42 & Forte \\
\hline $\begin{array}{l}\text { Fonte: Autora, } 2021 \\
\text { FR= Força de recomendação }\end{array}$ & \\
\hline
\end{tabular}

Tabela 5. intervençōes psicológicas e farmacológicas identificadas nos estudos

$$
\text { Lidocaína + prilocaína com hipnose }{ }^{25}
$$

\section{INTERVENÇÕES}

NÍVEL DA EVIDÊNCIA

FORÇA DE RECOMENDAÇÃO

$\oplus \oplus$

Forte

Forte al para avaliar a eficácia e foi notado uma redução significativa da ansiedade e dor 29.

A morfina foi testada e controlada por placebo nas crianças em oncologia pediátrica, os resultados mostraram que a morfina oral não proporcionou redução adicional de medo, angústia ou dor em comparação com placebo em pacientes pediátricos submetidos à inserção de agulha 42. Um estudo prospectivo controlado foi conduzido para comparar a eficácia de um anestésico local EMLA com uma combinação de EMLA com auto-hipnose. Os pacientes do grupo anestésico local associado à hipnose relataram menos ansiedade antecipada, e menos dor e ansiedade relacionada ao procedimento 25 .

Um estudo prospectivo randomizado e controlado de um protocolo de intervenção psicológica e farmacológica para o tratamento de leucemia pediátrica avaliou o sofrimento durante procedimentos invasivos na leucemia infantil. Mostraram diminuições ao longo do tempo na angústia e melhorias simultâneas na qualidade de vida e no estresse dos pais e apoiaram uma associação inversa entre angústia e idade da criança com a união de intervenções psicológicas e farmacológicas ${ }^{31}$.

\section{CONCLUSÃO}

A pesquisa respondeu à questão norteadora e objetivos, após apresentar que a assistência em oncologias pediátricas pode contar com intervenções farmacológicas e/ou comportamentais para reduzir sofrimento psíquico e dor. O caminho para a redução da dor e sofrimento psicológico é um processo complexo e pode haver um sinergismo entre intervenções psicológicas e farmacológicas. 


\section{Referências}

1. Maslak K, Favara-Scacco C, Barchitta M, et al. General anesthesia, conscious sedation, or nothing: Decision-making by children during painful procedures. Pediatr Blood Cancer. 2019;66(5):e27600. doi:10.1002/pbc.27600

2. INCA. Incidência de Cêncer no Brasil. Ministério da Saúde. 2020. chrome-extension:// ohfgljdgelakfkefopgklcohadegdpjf/https://www.inca.gov.br/sites/ufu. sti.inca.local/files/ media/document/estimativa-2020-incidencia-de-cancer-no-brasil.pdf.

3. Altounji $D, M c C l a n a h a n ~ R, ~ O ' B r i e n ~ R$, Murray P. Decreasing Central Line-Associated Bloodstream Infections Acquired in the Home Setting Among Pediatric Oncology Patients. J Pediatr Oncol Nurs. 2020;37(3):204-211. doi:10.1177/1043454220907551

4. Dupuis LL, Lu X, Mitchell HR, et al. Anxiety, pain, and nausea during the treatment of standard-risk childhood acute lymphoblastic leukemia: A prospective, longitudinal study from the Children's Oncology Group. Cancer. 2016;122(7):1116-1125. doi:10.1002/ cncr.29876

5. da Rosa dos Reis TL, Cardoso de Paula C, Potrich T, et al. Relações estabelecidas pelos profi ssionais de enfermagem no cuidado às crianças com doença oncológica avançada. Aquichan. 2014;14(4):496-508. doi:10.5294/aqui.2014.14.4.5

6. Kosir U, Bowes L, Taylor RM, et al. Psychological adaptation and recovery in youth with sarcoma: a qualitative study with practical implications for clinical care and research. BMJ Open. 2020;10(11):e038799. doi:10.1136/bmjopen-2020-038799

7. Wong A, Glogolia M, Lange PW, et al. A nurse-led paediatric oncology fast-track clinic proves a successful ambulatory intervention for patients. Support Care Cancer. 2020;28(9):4395-4403. doi:10.1007/s00520-019-05250-w

8. Taylor MR, Barton KS, Kingsley JM, Heunis J, Rosenberg AR. Defining a "Good Death" in Pediatric Oncology: A Mixed Methods Study of Healthcare Providers. Children. 2020;7(8):86. doi:10.3390/children7080086

9. A囚ikli E,Aydin Er R.Paediatric oncology patients' definitions of a good physician and good nurse. Nurs Ethics. November 2020:096973302096149. doi:10.1177/0969733020961499 10. Graetz D, Kaye EC, Garza M, et al. Qualitative Study of Pediatric Early Warning Systems' Impact on Interdisciplinary Communication in Two Pediatric Oncology Hospitals With Varying Resources. JCO Glob Oncol. 2020;6(6):1079-1086. doi:10.1200/go.20.00163 11. Clarke $M$, Horton R. Bringing it all together: Lancet-Cochrane collaborate on systematic reviews. Lancet. 2001;357(9270):1728. doi:10.1016/S0140-6736(00)04934-5

12. Moher D, Liberati A, Tetzlaff J, Altman DG. Preferred Reporting Items for Systematic Reviews and Meta-Analyses: The PRISMA Statement. PLoS Med. 2009:6(7):e1000097. doi:10.1371/journal.pmed.1000097

13. Souza PBM de, Ramos MDS, Pontes FAR, Silva SS da C. Coparenting: a study of systematic literature review. Estilos da Clin. 2016:21(3):700 doi:10.11606/issn.1981-1624. v21i3p $700-720$

14. Guyatt GH, Oxman AD, Vist GE, et al. GRADE: An emerging consensus on rating quality of evidence and strength of recommendations. BMJ. 2008:336(7650):924-926. doi:10.1136/bmj.39489.470347.ad

15. Higgins. Cochrane Handbook for Systematic Reviews of Interventions | Cochran Training. https://training.cochrane.org/cochrane-handbook-systematic-reviews-interventions. Published 2011. Accessed December 4, 2020

16. Kamsvåg T, Hedén L, von Essen L, Ljungman G. Ibuprofen in needle procedures in children with cancer-A feasibility and pilot study. Acta Paediatr Int J Paediatr. 2020. doi:10.1111/apa.15449

17. Haugen $M$, Skeens $M$, Hancock $D$, Ureda T, Arthur M, Hockenberry M. Implementing a pediatric oncology nursing multisite trial. J Spec Pediatr Nurs. 2020;25(3). doi:10.1111/ jspn.12293

18. Freedman $T$, Taddio A, Alderman $L$, et al. The cardTM system for improving the vaccination experience at school: Results of a small-scale implementation project on student symptoms. Paediatr Child Heal. 2019;24(Suppl 1):S42-S53. doi:10.1093/pch/pxz020

19. Mithal $P$, Simmons $P$, Cornelissen $T$, et al. To look or not to look during vaccination: $A$ pilot randomized trial. Can J Pain. 2018;2(1):1-8. doi:10.1080/24740527.2017.1412254

20. Yang $\mathrm{CH}$, Tian X, Yin H Bin, Gao XH, Li N. Sedation and analgesia with fentanyl and etomidate for intrathecal injection in childhood leukemia patients. Med (United States). 2015;94(1):e361. doi:10.1097/MD.0000000000000361

21. Hedén L, Von Essen L, Ljungman G. Effect of high-dose paracetamol on needle procedures in children with cancer-a RCT. Acta Paediatr Int J Paediatr. 2014;103(3):314-319. doi:10.1111/apa.12509

22. Lüllmann $B$, Leonhardt J, Metzelder $M$, et al. Pain reduction in children during port-à-cath catheter puncture using local anaesthesia with EMLATM. Eur J Pediatr. 2010;169(12):1465-1469. doi:10.1007/s00431-010-1244-1

23. Nguyen TN, Nilsson S, Hellström AL, Bengtson A. Music therapy to reduce pain and anxiety in children with cancer undergoing lumbar puncture: A randomized clinical trial. $J$ Pediatr Oncol Nurs. 2010;27(3):146-155. doi:10.1177/1043454209355983

24. Hedén L, Von Essen L, Frykholm P, Ljungman G. Low-dose oral midazolam reduces fear and distress during needle procedures in children with cancer. Pediatr Blood Cancer. 2009;53(7):1200-1204. doi:10.1002/pbc.22233

25. Liossi C, White P, Hatira P.A randomized clinical trial of a brief hypnosis intervention to control venepuncture-related pain of paediatric cancer patients. Pain. 2009;142(3):255263. doi:10.1016/j.pain.2009.01.017

26. Iannalfi A, Bernini G, Caprilli S, Lippi A, Tucci F, Messeri A. Painful procedures in children with cancer: Comparison of moderate sedation and general anesthesia for lumbar puncture and bone marrow aspiration. Pediatr Blood Cancer. 2005;45(7):933-938 doi:10.1002/pbc.20567

27. Sander Wint S, Eshelman D, Steele J, Guzzetta CE. Effects of distraction using virtual reality glasses during lumbar punctures in adolescents with cancer. Oncol Nurs Forum. 2002:29(1). doi:10.1188/02 onf.e8-e15

28. Ljungman $\mathrm{G}$, Gordh $\mathrm{T}$, Sörensen $\mathrm{S}$, Kreuger $\mathrm{A}$. Lumbar puncture in pediatric oncology: Conscious sedation vs. general anesthesia. Med Pediatr Oncol. 2001:36(3):372-379. doi:10.1002/mpo.1088

29. Ljungman $G$, Kreuger $A$, Andréasson $S$, Gordh T, Sörensen $S$. Midazolam nasal spray reduces procedural anxiety in children. Pediatrics. 2000:105(1 I):73-78. doi:10.1542/ peds.105.1.73

30. Liossi C, Hatira P. Clinical hypnosis versus cognitive behavioral training for pain management with pediatric cancer patients undergoing bone marrow aspirations. Int I Clin Exp Hypn. 1999;47(2):104-116. doi:10.1080/00207149908410025

31. Kazak AE, Penati $B$, Boyer $B A$, et al. A randomized controlled prospective outcome study of a psychological and pharmacological intervention protocol for procedural distress in pediatric leukemia. J Pediatr Psychol. 1996;21(5):615-631. doi:10.1093/ jpepsy/21.5.615

32. Jay S, Elliott $\mathrm{CH}$, Fitzgibbons I, Woody P, Siegel S. A comparative study of cognitive behavior therapy versus general anesthesia for painful medical procedures in children. Pain. 1995;62(1):3-9. doi:10.1016/0304-3959(94)00216-2

33. Mekonnen H, Gebreyohannis GT, Cherie A. Depression and associated factors among parents of children diagnosed with cancer at Tikur Anbessa Specialized Hospital, Addis Ababa, Ethiopia. Psychooncology. 2020;29(7):1141-1147. doi:10.1002/pon.5389

34. Gomberg J, Raviv A, Fenig E, Meiri N. Saving Costs for Hospitals Through Medical Clowning: A Study of Hospital Staff Perspectives on the Impact of the Medical Clown. Clin Med Insights Pediatr. 2020;14:117955652090937. doi:10.1177/1179556520909376 35. Chen CF, Huang FL, Chen YC, Wang IF, Huang YP. Maternal Caregiving for Children Newly Diagnosed with Acute Lymphoblastic Leukemia: Traditional Chinese Mothering as the Double-Edged Sword. J Pediatr Nurs. 2020;53:e64-e71. doi:10.1016/j. pedn. 2020.02 .008

36. Sullivan CE, Morrissey L, Day SW, Chen Y, Shirey M, Landier W. Predictors of Hospitals' Nonachievement of Baseline Nursing Standards for Pediatric Oncology. Cancer Nurs. 2020:43(4):E197-E206. doi:10.1097/NCC.0000000000000688

37. Graetz $D E$, Chen $Y$, Devidas $M$, et al. Interdisciplinary care of pediatric oncology patients in Central America and the Caribbean. Cancer. 2020. doi:10.1002/cncr.33339

38. Loeffen EAH, Mulder RL, Van De Wetering MD, et al. Current variations in childhood cancer supportive care in the Netherlands. Cancer. 2016;122(4):642-650. doi:10.1002 cncr.29799

39. Windich-Biermeier A, Sjoberg I, Dale JC, Eshelman D, Guzzetta CE. Effects of Distraction on Pain, Fear, and Distress During Venous Port Access and Venipuncture in Children and Adolescents With Cancer. J Pediatr Oncol Nurs. 2007;24(1):8-19. doi:10.1177/1043454206296018

40. Liossi C, White P, Hatira P. Randomized clinical trial of local anesthetic versus a combination of local anesthetic with self-hypnosis in the management of pediatric procedure-related pain. Heal Psychol. 2006;25(3):307-315. doi:10.1037/0278-6133.25.3.307

41. Wolitzky K, Fivush R, Zimand E, Hodges L, Rothbaum BO. Effectiveness of virtual reality distraction during a painful medical procedure in pediatric oncology patients. Psycho Heal. 2005;20(6):817-824. doi:10.1080/14768320500143339

42. Hedén LE, Von Essen L, Ljungman G. Effect of morphine in needle procedures in children with cancer. Eur J Pain. 2011;15(10):1056-1060. doi:10.1016/j.ejpain.2011.05.010

43. Tayeb BO, Eidelman A, Eidelman CL, Mcnicol ED, Carr DB. Topical anaesthetics for pain control during repair of dermal laceration. Cochrane Database Syst Rev. 2017:2017(2) doi:10.1002/14651858.CD005364.pub3 